\title{
Bayesian decision theoretic design of two-founder experimental crosses given diallel data
}

\author{
Gregory R. Keele ${ }^{*, \dagger}$, Paul L. Maurizio ${ }^{*, \dagger}$, Daniel Oreper ${ }^{*, \dagger}$ and William Valdar ${ }^{\dagger, \ddagger, 1}$ \\ ${ }^{*}$ Curriculum in Bioinformatics and Computational Biology, ${ }^{\dagger}$ Department of Genetics, ${ }^{\ddagger}$ and Lineberger Comprehensive Cancer Center, University of North \\ Carolina, Chapel Hill, North Carolina 27599 \\ ORCID IDs: 0000-0002-1843-7900 (G.R.K.), 0000-0002-5859-6260 (P.L.M.), 0000-0003-4569-5160 (D.O.), 0000-0002-2419-0430 (W.V.)
}

\begin{abstract}
In designing experimental crosses of inbred strains of model organisms, researchers must make a number of decisions. These include the selection of the appropriate strains, the cross design (eg. F2 intercross), and the number of progeny to collect (sample size). These decisions strongly influence the potential for a successful quantitative trait locus (QTL) mapping experiment; good design decisions will lead to efficient and effective science. Thus experimental design deserves careful consideration and planning. Experimental outcomes can be quantified through utility functions using a Bayesian decision theoretic approaches. For QTL mapping experiments, the power to map a QTL is an appealing utility function to maximize. Using any utility function to aid in experimental design will be dependent on assumptions, such as the QTL effect size in the case of power. Rather than arbitrarily selecting QTL effect size values, they can be estimated from pilot data using a Bayesian hierarchical model. The information in the pilot data can be propagated to the utility function, using Markov Chain Monte Carlo $(\mathrm{MCMC})$ to sample from the posterior distribution. Key features of this approach include: 1) distributional summaries of utility, which are preferable to point estimates, and 2) a comprehensive search of the experimental space of crosses of inbred lines for well-designed experiments. We evaluate this Bayesian theoretic approach using diallel crosses as the pilot data. We present results from simulations as well as present examples from both Mendelian and complex traits in the founder strains of the mouse Collaborative Cross. All analyses were performed using our R package, DIDACT (Diallel-Informed Decision theoretic Approach for Crosses Tool), developed to perform Bayesian cross selection based on diallel pilot data.
\end{abstract}

KEYWORDS BayesDiallel, F2 intercross, backcross, utility, complex trait

\section{Introduction}

Geneticists commonly conduct experiments with the goal of identifying quantitative trait loci (QTL) using crosses of inbred lines of model organisms. These experiments can be costly in terms of resources, due to the organisms, their care, genotyping or sequencing, as well as the time and energy required for the experiment itself. In the face of these constraints, procedures that explore the potential set of experimental cross designs and allow researchers to select experiments with greater potential to be successful are beneficial to the field of complex traits.

Manuscript compiled: Thursday $6^{\text {th }}$ December, 2018

${ }^{1}$ Corresponding author: 120 Mason Farm Rd., Genetic Medicine Bldg., Suite 5022,

Campus Box 7264, University of North Carolina, Chapel Hill, NC 27599.

E-mail: william.valdar@unc.edu
Although the goals for a given experiment will be nuanced and unique to each study, the mapping portion is successful if a QTL is detected with a statistically significant signal, using established methodologies (Lander and Botstein 1989; Haley and Knott 1992; Dupuis and Siegmund 1999; Broman 2001). This outcome is not guaranteed simply due to the presence of segregating QTL in the mapping population: the experimental design may not be sufficiently powered to identify them. The power of an experiment, the probability that a non-zero effect will be recognized given that it is present, is influenced by a number of biological factors, some of which can be more easily manipulated and optimized through experimental design choices. These factors include genetic architecture, mode of action, and the variation in the population due to noise. If the genetic architecture of the trait is highly polygenic with many loci of small effect, power will be reduced compared to tests for QTL of larger effect. 

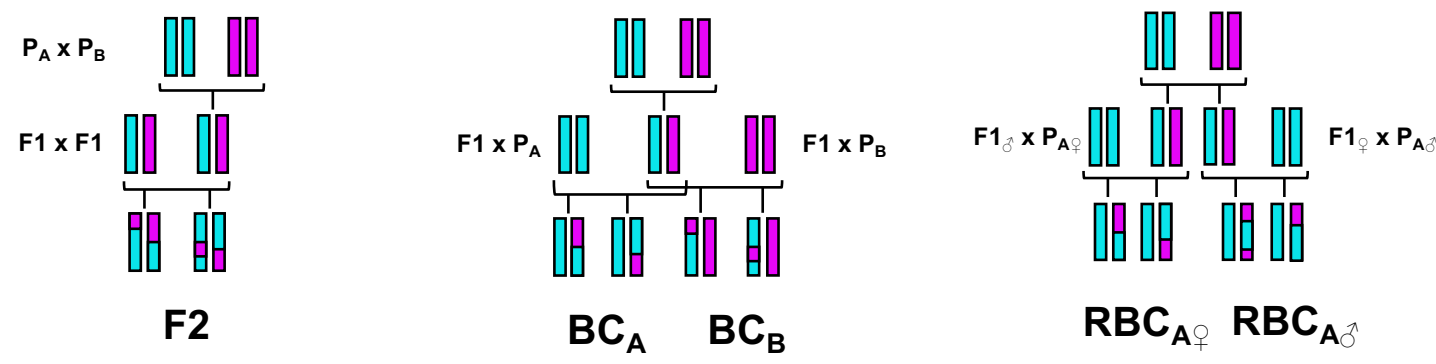

Figure 1 The potential bi-parental crosses considered within DIDACT: F2 [left], BC [middle], and RBC ${ }^{\mathrm{PO}}$ [right]. A single inbred genome is represented as a colored chromosome. The parental $(P)$ and $F 1$ generations are replicable, whereas the mapping populations are not. All three genotypes at a locus ( $\mathrm{A} / \mathrm{A}, \mathrm{A} / \mathrm{B}$, and $\mathrm{B} / \mathrm{B})$ can be observed in the $\mathrm{F} 2$ population, allowing for the estimation of additive and dominance effects for a putative QTL. With traditional BC, only the homozygote of the backcrossed parental allele is observed. By jointly analyzing $\mathrm{RBC}^{\mathrm{PO}}$, it is possible to detect effects from heterozygous sites in which the parent-of-origin differs for the backcrossed parental allele.

Similarly, mode of action (e.g. additive, dominant), for a QTL will also influence power because certain experimental designs will have differential ability to detect a given effect type. For example, a backcross (BC) cannot identify a QTL with a fully recessive effect when the homozygote of the recessive allele is never observed. Finally, an increase in variation due to noise will decrease power because the noise drowns out the true signal. Ideally, investigators would select the experiment that can best handle these factors in the given setting.

In the context of crosses of inbred organisms, one major component of the experimental design is the founder or parental lines. The selection of parental lines allows the investigator to control the genetic background of the experimental population, which can greatly influence the previously mentioned biological factors, and ultimately influence the potential for mapping success. For example, a trait could be highly polygenic and have loci with complex modes of action within natural populations, but much of the genetic and phenotypic variation becomes fixed within two closely related inbred lines. The reduced genetic variability can impact all of the biological factors: the complexity or polygenic nature of the genetic architecture by fixing many of the loci, the mode of action by limiting the potential for epistatic effects through less segregating variants, and the variance attributable to noise through the reduction in phenotypic variability.

The ability to strongly influence the sources of variation in the population is important to consider. If the QTL explains a large proportion of the variance in the population, a simple cross will be well-powered to identify the QTL, even if its effect is small. The balance between the variance attributable to the QTL versus how generalizable the experiment is to natural populations is important to consider when making decisions about experimental design. Ultimately a finding that is characteristic of only a very unnatural experimental population and does not generalize well to more natural ones, will greatly reduce the impact of such an experiment and even undermine the purpose of experiments with model organism in general. The ideal experiment will be well-powered to identify QTL, but also generalizable to natural populations.

The power of an experiment cannot be directly assessed because it requires knowledge of the true effect, which is unknown. Instead power calculations are performed for a range of plausible parameters, usually over varying effect sizes or sample sizes, given some type I error level and error variance, which can then be represented as power curves. Analytical solutions to power calculations have been specifically developed and refined for simple cross designs such as F2 intercross, BC, and recombinant inbred lines (RIL) panels, using an information perspective approach, which posits that the complete information is composed of the unobserved information and the missing information (Sen et al. 2005). These power estimates are still dependent on assumed parameters, in this case QTL effect sizes and error variances. As a result, meaningful and useful power calculations still depend on the consideration of an appropriate set of values for these unknown quantities, otherwise the power estimates could be uninformative or even misleading.

Pilot data can provide information about the underlying genetic signals present in potential experiments. One source of pilot data is the inbred founder lines themselves as well as their hybrid crosses (F1). Comparisons of F1 individuals to the inbred strains can provide estimates of various genetic effects for given lines, aggregated from causal variants across the entire genome. These effects can include additive, inbred, and epistatic. An additive effect for a given strain can be estimated from averages of $\mathrm{F} 1$ that do not have the strain as a parent ( 0 copies), to averages of F1 that do have the strain as a parent (1 copy), and finally to the inbred line itself ( 2 copies). An inbred effect is estimated from these same sets of crosses, but represent the average departures observed from the expectation of the hybrid according to the additive effect to its actual observed value. An epistatic effect represents departures from expectation for a specific cross of two strains, thus it is an interaction of the two strains.

Additional information is contained in the reciprocal crosses that compose the F1 hybrids, and can be characterized as parentof-origin effects (POE). Reciprocal F1 crosses have the same parental lines, but the dam-sire identities are switched. Average differences between reciprocal crosses can be used to estimate POE, allowing QTL that underly these POE to be mapped using a unique $\mathrm{BC}$ design that we will refer to as reciprocal parentof-origin ( $\left(\mathrm{RBC}^{\mathrm{PO}}\right.$ ) (Gonzalo et al. 2007). Traditional reciprocal $\mathrm{BC}$ refer to related $\mathrm{BC}$ in which $\mathrm{F} 1$ are back-crossed to alternative parental lines, whereas $\mathrm{RBC}^{\mathrm{PO}}$ have the same F1 and back-crossed parent, but the dam and sire strains are reversed between reciprocal pairs; thus the parent-of-origin for each allele is known at heterozygous sites, and differences in the trait that correlate to genotype and parent-of-origin can be detected. The estimation of POE through reciprocal crosses allows researchers to add RBC to their collection of potential experiments. Though $\mathrm{RBC}^{\mathrm{PO}}$ are not as frequently used as $\mathrm{F} 2$ and $\mathrm{BC}$, interest in $\mathrm{POE}$ has increased (Lawson et al. 2013; Bérénos et al. 2014; Connolly 
and Heron 2015; Harper et al. 2014; Zou et al. 2014). Pilot data that distinguish between reciprocal F1s allow for an even larger number of experiments to be explored and considered. These potential bi-parental mapping populations, $\mathrm{F} 2, \mathrm{BC}$, and $\mathrm{RBC}^{\mathrm{PO}}$, are depicted in Figure 1.

These experiments can best be explored with the full set of potential founder lines and their F1 hybrids, which represent a classic genetic experiment, the diallel cross. Diallels have been used to study numerous traits in a diverse range of organisms, including: mating speed, female receptivity, and temperature preference in flies (Parsons 1964; Casares et al. 1992; Yamamoto 1994); immune function, polyandry, and genetic-environment interactions in crickets (Rantala and Roff 2006; Ivy 2007; Nystrand et al. 2011); and heterosis and reciprocal effects in poultry (Fairfull et al. 1983). Additionally, the diallel has a long history in plant breeding (Gilbert 1958) and numerous recent applications (Bahari et al. 2012; Ghareeb Zeinab and Helal 2014; Dos Santos et al. 2016).

Since being described in the early $20^{\text {th }}$ century, statistical methodology for the diallel has seen steady advancements, from estimating the general combining ability of related F2 populations (Griffing 1956) to random effect (Zhu and Weir 1996; Tsaih et al. 2005) and Bayesian hierarchical modeling of sparse diallel (Greenberg et al. 2010). Recently, Lenarcic et al. (2012) used Bayesian hierarchical modeling of diallel data to allow for stable estimation of a large number of strain-level genetic effects (such as additive, inbred, epistatic, and maternal), and this method has been used to analyze a number of phenotypes and organisms, such as cranial shape (Gonzalez et al. 2016), response to treatment and infection (Crowley et al. 2014; Maurizio et al. 2018), and litter size (Shorter et al. 2018) in mice, and shoot growth in carrots (Turner et al. 2018). Even incomplete or sparse diallel data can be used for the characterization of some of the underlying strain-level genetic signals, which can then be used to evaluate the potential space of experiments, and potentially allow for the selection of a favorable one. A simplified representation of a diallel, in the founders of the Collaborative Cross (CC), a multiparental recombinant inbred panel in laboratory mouse, is depicted in Figure 2.

A number QTL mapping approaches have been developed within the context of jointly modeling related populations, including daughter populations of a diallel cross (Rebaï and Goffinet 1993, 2000; Liu and Zeng 2000; Li et al. 2013), as well as QTL allele effect estimation for these related populations (Jannink and Wu 2003). More recently, Verhoeven et al. (2006) investigated jointly modeling diallel data with the related downstream F2 populations, and found that it allowed for the simultaneous dissection of the trait across all the populations, or characterization of strain-level effects, as well as generalization of the QTL findings from the mapping populations in terms of the multiparental diallel population. We focus on the situation in which none of the F2 populations, or any such downstream cross populations, are observed, and attempt to evaluate the utility of potential crosses in terms of QTL mapping. Herein we bring together three lines of research:

1. The estimation of the power to map putative QTL of given effect sizes.

2. The characterization of strain-level genetic effects from diallel pilot data.

3. The selection of optimal experiments through a decision theoretic approach.

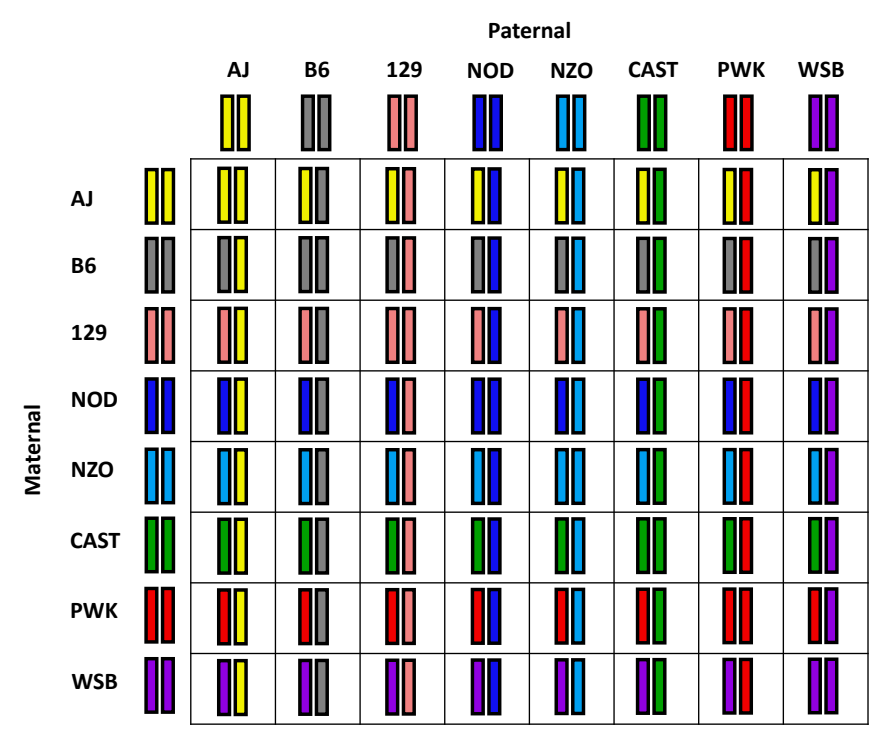

Figure 2 A diallel of the $\mathrm{CC}$ founders. Each unique strain genome is represented as a colored chromosome. Genomes along the diagonal represent the inbred founders. Offdiagonal genomes represent the F1 hybrids of a pair of founders. Reciprocal F1 genomes possess the same genome, ignoring mitochondria and sex chromosomes for males, but parent-of-origin for each chromosome will be reversed. All the genomes of an inbred diallel cross are replicable, and thus replicate observations can be measured per genome. Some cells of the diallel may not be observed, reducing the ability to accurately estimate certain strain-level effects.

We use the BayesDiallel model (Lenarcic et al. 2012), a Bayesian hierarchical model for characterizing the genetic information contained in diallel data as aggregate strain effects. Bayesian approaches can stably estimate a large number of genetic effects through the sharing of information across strains, as well as assess the uncertainty around these effects. These strain effects are next propagated to utility functions, including power to map a putative QTL underlying the strain effects, for an array of potential experimental crosses. Our approach will allow researchers to select better experiments with greater potential based on pilot data over ineffective or inefficient options. These opportunities include not only favorable experiments for mapping additive traits, which have commonly been studied, but also for mapping the QTL responsible for less well-understood effects such as POE.

\section{Statistical Models and Methods}

Our approach builds on three separate areas of research. Firstly we consider the calculation of power to map QTL given that the QTL effect $\theta$ is known. This will require the review of general concepts in quantitative genetics and statistics in the context of crosses of two inbred lines. Because in reality $\theta$ is never actually observed, we next consider the characterization of $\theta$ from pilot data. Finally we discuss the selection of optimal experimental crosses through the maximization of a chosen utility function.

\section{Power to map QTL}

Single QTL model. Here we review the general concepts in quantitative genetics and statistics that support the method used by 
Sen et al. (2005) for power calculations of traditional crosses such as the F2 and BC. Consider this model:

$$
y_{i}=\mathrm{QTL}_{i}+G_{i}+E_{i}+\epsilon_{i}
$$

where $y_{i}$ is the phenotype of individual $i$, QTL $L_{i}$ is the effect of the QTL for individual $i, G_{i}$ is the effect of other genetic elements for individual $i, E_{i}$ is the effect of environmental factors for individual $i$, and $\epsilon_{i}$ is the random noise for individual $i . G_{i}$ and $E_{i}$ are un-modeled, and can thus be collapsed with $\epsilon_{i}$ into a single error term $\varepsilon_{i}$.

$$
y_{i}=\mathrm{QTL}_{i}+\varepsilon_{i}
$$

where $\varepsilon_{i} \sim \mathrm{N}\left(0, \sigma^{2}\right)$ with $\sigma^{2}$ representing the error variance in the data. The QTL effect is a vector, traditionally parameterized as additive and dominant effects (Lynch and Walsh 1998). This can be formulated in a traditional regression framework:

$$
\begin{aligned}
\mathbf{y} & =\mathbf{X} \boldsymbol{\beta}+\boldsymbol{\varepsilon} \\
& =\mathbf{X}\left[\begin{array}{l}
\mu \\
\alpha \\
\delta
\end{array}\right]+\boldsymbol{\varepsilon},
\end{aligned}
$$

where $\mathbf{y}$ is the phenotype vector, $\mathbf{X}$ is the design matrix that we will define further, $\beta$ is the vector of effects composed of $\mu$, the overall phenotypic mean, $\alpha$, the additive effect of the QTL, and $\delta$, the dominance effect for the QTL, and $\varepsilon$ is the vector of errors. Consider an $\mathrm{F} 2$ or BC of strains $A$ and $B$, with the genotype of an individual represented in terms of strain identity, denoted in the subscript. $\alpha$ is the midpoint of the difference between the homozygotes:

$$
\alpha=\frac{\mathrm{E}\left(y_{A A}\right)-\mathrm{E}\left(y_{B B}\right)}{2}
$$

$\delta$ is the deviation of the heterozygote from the average of the homozygotes:

$$
\delta=\mathrm{E}\left(y_{A B}\right)-\frac{\mathrm{E}\left(y_{A A}\right)+\mathrm{E}\left(y_{B B}\right)}{2}
$$

Table S1 lists Eq 3 parameterized in terms of these QTL effects. This parameterization maintains the identifiability of all the effects, though it may not be as intuitive to researchers accustomed to more traditional regression models used commonly in genome-wide association studies.

Returning to the formulation of the model in Eq 6, the variance of the model can be characterized as follows with the assumption that there is no covariance between the QTL effect and the error,

$$
\operatorname{Var}(y)=\operatorname{Var}(\mathrm{QTL})+\sigma^{2}
$$

The background genetic and environmental variation are captured in $\sigma^{2}$; here we focus on the variability due to the QTL. E $(y)$ will vary depending on the genotype, which will vary probabilistically according to the type of cross, as described in Table S1. As example, for an $\mathrm{F} 2$ cross, the $\operatorname{Pr}(A A)=\frac{1}{4}, \operatorname{Pr}(A B)=\frac{1}{2}$, and $\operatorname{Pr}(B B)=\frac{1}{4}$. The variance of a random variable $X$ is defined as $\operatorname{Var}(X)=\mathrm{E}(X-\mathrm{E}(X))^{2}$. The variable $X$ in this setting is QTL, which is the categorical genetic state at the QTL. The expectation of $X$ is $\mathrm{E}(X)=\sum_{x \in \mathcal{X}} x \operatorname{Pr}(X=x)$. Based on the genotype probability for a given cross, the variances due to the QTL in terms of the QTL effects are presented in Table S2.

The mode of action of the locus impacts the variability in phenotype due to QTL within a cross type, as seen in Table
S2. This is particularly noticeable in the BC experiments, where certain modes of action produce no variance. If the locus is recessive (or conversely dominant), the genotype with differing phenotype will not be observed, and nor will variation due to QTL. Finally, cross type also impacts the QTL variance, which is also clear in Table S2. Increasing the variance attributable to the QTL will increase power to map the QTL; in contrast, increasing the overall variance that is attributable to noise (un-modeled background genetic factors or environmental factors) will reduce the significance of statistical tests, and thus decrease the power.

Power calculations. Analytical power calculations are the probability mass above some threshold for the distribution of a statistic of interest under the alternative hypothesis. This requires that the alternative distribution be reasonably characterized. Consider $\theta$, some function of the QTL effects $\alpha$ and $\delta$, as the parameter of interest. We wish to calculate the probability of mapping the QTL that results in $\theta$. In terms of the association modeling, a natural null hypothesis is $H_{0}: \theta=\theta_{0}$ with $\theta_{0}=0$, that there is no QTL effect. The alternative hypothesis is $H_{A}: \theta \neq \theta_{0}$. By specifying a model for the data, or more precisely the distribution of the error term of the model, the likelihood $\mathcal{L}(\theta)$ can be evaluated. The likelihood ratio test (LRT) statistic, $T=-2 \log \frac{\mathcal{L}(\theta=0)}{\mathcal{L}(\theta=\widehat{\theta})}$, where $\widehat{\theta}$ is a proposed estimate of $\theta$, can be used to perform power calculations.

To use the LRT statistic for power calculations, a significance threshold and corresponding statistic distribution for $T$ are necessary. The traditional scale of significance used in the linkage and QTL fields is the $\log _{10}$ likelihood ratio or LOD (logarithm of odds) score. Historically a LOD score of $3(2 \log (10) \times 3$ on the likelihood ratio scale) has been used as a significance threshold, meaning approximately that the data support the alternative model over the null model 1000 to 1 . A more stringent significance threshold than 3 can be used to further reduce the risk of false positives or possibly account for a multitude of tests (though it is worth noting these tests will not be fully independent). Given some significance threshold $C$ is chosen to determine genome-wide significance; if $T \geq C$ for some locus, the null hypothesis is rejected. The threshold $C$ will affect the the true positive and false positive rates, and more important to our topic, the power.

Statistically, power is the probability that the null hypothesis is rejected given that alternative hypothesis is true. The LRT T is the statistic upon which the power calculations are drawn, thus the power will be $\operatorname{Pr}\left(T \geq t \mid \theta \neq \theta_{0}\right)$ where $t$ is the observed statistic produced by the data. With the LRT statistic, when the models are nested and the maximum likelihood estimate (MLE) is used $\left(H_{A}: \theta=\widehat{\theta}_{\mathrm{MLE}}\right)$, as they are in this case, and the null model is true, $T$ is asymptotically $\chi_{k}^{2}$ distributed, where $k$ is the degrees of freedom, the difference in number of parameters between the models. A power calculation from this distribution would not be useful because it would represent the probability that the null hypothesis is rejected when there is no genetic effect, or the false positive probability. The power is rather based on the alternative hypothesis being true, $\theta \neq \theta_{0}$, and thus $\chi_{k}^{2}$ distribution is inappropriate. When the alternative hypothesis is true rather than the null, that $\theta=\widehat{\theta}_{\mathrm{MLE}}, T$ is proportional to the noncentral $\chi^{2}$ distribution with noncentrality parameter $\left(\theta-\theta_{0}\right)^{T} \mathcal{I}(\theta)\left(\theta-\theta_{0}\right)$ where $\mathcal{I}(\theta)$ is the expected Fisher information matrix. We model the data with a Gaussian mixture distribution with a shared residual variance, which naturally extends from the two line cross statistical model. A key feature 
of this model is that the LRT reduces to the variance attributable to the QTL as a function of effects that we presented in table 1. This variance parameter is scaled by $\sigma^{2}$, which sets the variance of each Gaussian component to 1 . Thus the power calculations are intuitively a function of the effect size, the proportion of the variance explained by the QTL (effect size combined with residual error variance), and the sample size.

It is important to note that the actual $\widehat{\theta}_{\text {MLE }}$ cannot be calculated because no actual cross data for QTL mapping is observed, but the underlying theory of the method assumes that the alternative $\theta$ is the MLE estimator. $\sigma^{2}$ is also never actually known, but we estimate it from the information present in the pilot data. The final interpretation of this power calculation is the probability that a significant result is found $(T \geq t)$ given that there is some QTL effect specified in the proposed MLE estimator $\widehat{\theta}_{\text {MLE }}$ with an error variance of $\sigma^{2}$.

Sen et al. (2005) develop the theory further to account for the fact that the information is generally never complete in QTL studies. The true QTL variant is most likely not observed (genotyped), but rather loci in linkage disequilibrium are, and thus contain some of the information from the QTL. They develop the theory to take into account the missing information from sparse markers (as previously described), as well as selective genotyping (genotyping study individuals on the tails of the phenotype distribution). As a result of this, power can be reduced by not only greater error variance, but also missing information. The advancement in genotyping technology is generally leading to denser markers in QTL studies, leading us to make the assumption of complete information. We directly incorporate the $\mathrm{R}$ package qtlDesign (Sen et al. 2007) into our method, so missing information can be specified in the power calculations. See Sen et al. (2005) for a description of the missing information theory used.

\section{Strain-level genetic effects}

The power calculations described above are dependent on known QTL effects $\theta$, but in reality, $\theta$ is not observed. However, information about $\theta$ is contained in pilot data, which can be exploited to characterize plausible distributions for $\theta$.

Bayesian modeling of diallel data. One potential convenient source of pilot data are the parental lines and some subset of their F1 hybrids. Direct estimation of $\theta$ is not possible because no recombinations occur between the parental haplotypes within F1 individuals, but rather strain effects that represent the accumulated effect of the segregating variants within each inbred strain can be estimated. Denote these strain effects, the vector of effects that will be defined in $\mathrm{Eq} 7$, as $\phi$ to distinguish them from $\theta$, the effect of a single QTL.

The strain-level vector $\boldsymbol{\phi}$ can encompass effects of different modes of actions based on the strain identities of the dam and sire of an individual. These strain-level effects include additive, inbred, epistatic, and maternal. The additive effects characterize the average effect of a strain constrained to a dosage-like model. Such a simple model is not always sufficient to accurately model data, such as the situation that an F1 hybrid is not approximately the midpoint between the parental strain phenotypes. We account for this potential deviation from additivity with an inbred effect, which is in contrast to the more traditional view of nonadditivity as dominance. This parameterization of the model is appropriate for our pilot data because, considering $J$ parental strains, there will be $J(J-1)$ possible F1 hybrids, and only $J$ inbreds. When $J$ is greater than 2, which is likely, the number of possible hybrid F1 will outnumber the $J$ lines. Thus modeling the state of being outbred as the default state more intuitively matches the structure of our data.

Epistatic and maternal effects represent other potential sources of deviation from strict additivity. Epistatic effects are essentially an interaction between strains, thus allowing a specific F1 hybrid to deviate from its additive expectations. Maternal effects can capture strain-specific POEs where there is an average difference between reciprocal F1. As demonstrated in Lenarcic et al. (2012), consider pilot data that are some subset of the $J$ inbred strains and their F1. The strain identities of dam, sire, and dam-sire pair for individual $i$ are indexed as $j[i], k[i]$, and $(j, k)[i]$, respectively. We model the pilot data as

$$
\begin{aligned}
y_{i}=\mu & +\underbrace{a_{j[i]}+a_{k[i]}}_{\text {additive }}+\underbrace{I_{\{j=k\}}\left(b_{j}+\beta_{\text {inbred }}\right)}_{\text {inbred }} \\
& +\underbrace{I_{\{j \neq k\}}\left(v_{(j, k)[i]}+w_{(j, k)[i]}\right)}_{\text {epistatic }}+\underbrace{m_{j[i]}-m_{k[i]}}_{\text {maternal }}+\varepsilon_{i},
\end{aligned}
$$

where $y$ is the continuous phenotype value, $\mu$ is the intercept, $a$ is a strain-specific additive or dose effect, $\beta_{\text {inbred }}$ and $b$ are respectively a general inbred effect and a strain-specific inbred effect that are included only if individual $i$ is inbred, $v$ and $w$ are respectively symmetric and asymmetric strain-by-strain interaction effects that we will refer to as epistatic effects and are only included if individual $i$ is a hybrid, $m$ is a strain-specific maternal effect, and $\varepsilon_{i}$ is the individual-specific noise (deviation from the model expectation) and is distributed: $\varepsilon_{i} \sim \mathrm{N}\left(0, \sigma^{2}\right)$. The model can also include important covariates, such as sex, that need to be adjusted for as fixed effects. The complete set of founder strains and all their reciprocal F1 hybrids represent what is called a diallel, which would allow for the estimation of the full set of strain effects described. Although an incomplete diallel cannot estimate all the strain effects, it still provides information that can be used to estimate $\boldsymbol{\phi}$. See Appendix A for descriptions of prior specifications used.

Connecting strain-level effects to QTL effects. Transitioning from strain-level genetic effects $\phi$ to the effect of a single QTL $\theta$ requires some strong assumptions. Pilot data consisting solely of F1 individuals cannot provide information about specific loci or the number of loci contributing to a strain effect; there are an infinite number of genetic architectures that can explain a given strain effect. It is possible that conducting a small set of F2 crosses and investigating the variability in phenotype for the resulting population could provide information about the trait genetic architecture, such as distinguishing between highly polygenic and oligogenic traits, but here we focus on using only the F1 generation. We make the assumption that the strain effects represent the effect of a single QTL, which is biologically unlikely but provides an informed approach to connect information in the pilot data to the power calculations. Eq 7 provides expected phenotype values for a given cross of two strains, assuming the trait is controlled by a single QTL. Consider comparing strains $A$ and $B$. Eq 7 can be used to estimate $\mathrm{E}\left(y_{A A}\right), \mathrm{E}\left(y_{A B}\right)$, and $\mathrm{E}\left(y_{B B}\right)$. From these predicted values, traditional single QTL additive $(\alpha)$ and dominant effects $\delta$ can be estimated from Eq 4 and Eq 5. These estimates along with estimates of $\sigma^{2}$ can then be used with the power calculation machinery described before. Different QTL effects will be estimated from the model in Eq 7 for different potential crosses of inbred lines. 
Generalizing single QTL effect for complex traits. The QTL effects defined in Eq 4 and Eq 5 make the assumption of a single underlying QTL; however, from the perspective of the strainlevel model in Eq 7, these parameters can be interpreted as strain-level predicted phenotype contrasts. The corresponding variance expressions in Table $\mathbf{S} 2$ then become simplifying functions of contrasts. Generally speaking, all of these quantities can be viewed as point summaries of predicted contrasts from a strain-level model, and thus have a meaningful and potentially useful interpretation outside of the single QTL Mendelian context.

\section{Decision theoretic approach}

Different inbred lines will possess differing segregating variants to potentially identify. We use our model of pilot data to make predictions for some set of possible experiments, which can be viewed in the context of a decision theoretic space (Raiffa and Schlaifer 2000). Define $\mathcal{A}$ as the set potential experimental crosses. Considering $n$ inbred lines, $\mathcal{A}$ could contain all of or some subset of the $\left(\begin{array}{l}n \\ 2\end{array}\right)$ potential F2 crosses and $3 n(n-1)$ potential BC.

Power as utility function. Let an element of $\mathcal{A}$, a represent a specific action, in this setting, a cross experiment that has corresponding single QTL effect composed of $\alpha$ and $\delta$. If we define $Q$ to be a binary variable that the QTL that causes $\theta(\alpha$ and $\delta)$ is successfully mapped:

$$
Q: \begin{cases}q=1 & \text { QTL is mapped } \\ q=0 & \text { QTL is not mapped }\end{cases}
$$

$\operatorname{Pr}(Q=1 \mid \mathfrak{a})$ represents the power that the QTL is successfully mapped, and can be calculated using the noncentral $\chi^{2}$ distribution described previously. We next define $\mathcal{C}$ to be the consequence or experiment outcome space for a QTL mapping experiment, where $c=\left\{q_{1}, \ldots q_{p}\right\}$ is the specific joint mapping outcome of the $p$ QTL that underly the strain effects. Along with using posterior contrasts as the utility function, this approach allows for the assumption of a single QTL causing the strain-level effects to be reduced.

A utility function is an important concept in decision theory. It provides a common scale to compare potential experimental outcomes, and select optimal experiments. Alternative utility functions can be devised and easily swapped to place value on differing aspects that investigator want to prioritize. We define a utility function, $u($.$) , to map from \mathcal{C}$ to the reduced utility space, $\mathcal{U}$, which we pose as a function of power, a natural quantity to prioritize. Consider the probability of a specific consequence, which will be a product of a function of the individual power for each QTL: $\operatorname{Pr}\left(c=\left\{q_{1}, \ldots, q_{p}\right\} \mid \mathfrak{a}\right)=\prod_{i=1}^{p} \operatorname{Pr}\left(Q_{i}=q_{i} \mid \mathfrak{a}\right)$. We define $u($.$) to be the count of p$ QTL that were successfully mapped: $u(c)=\sum_{i=1}^{p} q_{i}$. The probability of a utility $v$ can be calculated from subsets of $\mathcal{C}$ :

$$
\begin{aligned}
\operatorname{Pr}(v \mid \mathfrak{a}) & =\sum_{c \in \mathcal{C}: v=u(c)} \operatorname{Pr}(c \mid \mathfrak{a}) \\
& =\sum_{c \in \mathcal{C}} I_{\{v=u(c)\}} \operatorname{Pr}(c \mid \mathfrak{a})
\end{aligned}
$$

Strictly speaking, the probability of a utility is also dependent on QTL effect $\theta: \operatorname{Pr}(v \mid \mathfrak{a}, \theta)=\sum_{c \in \mathcal{C}} I_{\{v=u(c)\}} \operatorname{Pr}(c \mid \mathfrak{a}, \theta)$. $\theta$ can be marginalized out through integration: $\operatorname{Pr}(v \mid \mathfrak{a})=$ $\int_{\theta} \operatorname{Pr}(v \mid \mathfrak{a}, \theta) \operatorname{Pr}(\theta \mid \mathcal{D}) d \theta$, where $\mathcal{D}$ represents the pilot data.The probability of this utility function provides an evaluation of the uncertainty of mapping QTL of a given effect size, but does not take into account the uncertainty of $\alpha, \delta$, and $\sigma^{2}$, which are produced from the Bayesian model. Through Gibbs sampling or some other Markov Chain Monte Carlo (MCMC) method, a Bayesian model can produce $S$ draws from the posterior distribution of these parameters. Monte Carlo (MC) averaging allows us to take into account this extra source of variability, resulting in the posterior expected utility for cross $\mathfrak{a}$ :

$$
\begin{aligned}
\operatorname{PEU}(\mathfrak{a}) & =\int_{\theta} \int_{v} v \operatorname{Pr}(v \mid \mathfrak{a}, \theta) d v d \theta \\
& =\int_{\theta} \sum_{v \in \mathcal{U}} v \sum_{c \in \mathcal{C}} I_{\{v=u(c)\}} \operatorname{Pr}(c \mid \mathfrak{a}, \theta) d \theta
\end{aligned}
$$

where $\theta$ is the vector function of $\alpha, \delta$, and $\sigma^{2}$. The quantity $\sum_{v \in \mathcal{U}} v \operatorname{Pr}(v \mid \mathfrak{a}, \theta)$ within the $\operatorname{PEU}(\mathfrak{a})$ is the expected utility for a single draw $s$ from the Bayesian model. This quantity is then averaged over the QTL effect space of the posterior distribution, traversed through the MC samples. This can be summarized as a point estimate such as the posterior mean or median, or the posterior distribution of expected utilities can be plotted for a given cross $\mathfrak{a}$. Interpretations of the $\operatorname{PEU}(\mathfrak{a})$ will vary amongst utility functions, but we will focus our discussions on power as the utility being maximized.

If it is assumed that all $p$ QTL have the same effect size, the utility function $u(c)$, the number of $p$ QTL that were successfully mapped, follows a binomial distribution. Consider simple case of a single QTL $(p=1)$, in which the binomial reduces to the Bernoulli distribution. In this setting, the PEU $(\mathfrak{a})$ reduces to the posterior probability of mapping the QTL. When $p$ is greater than one, as with a binomial variable, $\operatorname{PEU}(\mathfrak{a})$ now represents the expected number of QTL to be mapped. Our approach should be flexible to any reasonable utility function investigators can define, but we emphasize power because its $\operatorname{PEU}(\mathfrak{a})$ are easy to interpret, as well as posterior contrasts, which have require less assumptions and better extend to highly complex phenotypes.

\section{Simulation of diallel data}

Diallel data were simulated from Eq 7 based on a number of different strain-level effect settings to assess how well DIDACT performs in a variety of genetic architectures according to various quantities, such as the estimation of the strain-level effects, and more importantly, utility. Specifically 100 realizations were simulated of a full and balanced diallel of eight strains with five individuals per diallel cell, resulting in a total of $320 \mathrm{ob}-$ servations, for each specification of strain-level effects. The strain-level effects were scaled so that the proportion of total variance was controlled such that:

$$
\operatorname{var}(\boldsymbol{a})+\operatorname{var}(\boldsymbol{b})+\operatorname{var}(\boldsymbol{v})+\operatorname{var}(\boldsymbol{w})+\operatorname{var}(\boldsymbol{m})+\operatorname{var}(\boldsymbol{\varepsilon})=1
$$

The proportion of variance from strain-level effects was set to 50 evenly spaced values between 0.01 to 0.99 . The following strain-level effect specifications were used:

1. biallelic_add: The inbred, maternal, and both epistatic effects are all set to 0 . Two alleles are simulated for the additive effects and distributed evenly amongst the parental strains.

2. rnorm_add: The inbred, maternal, and both epistatic effects are all set to 0 . The eight additive alleles are simulated such that $a_{j} \sim \mathrm{N}(0,1)$. 
bioRxiv preprint doi: https://doi.org/10.1101/489682; this version posted December 7, 2018. The copyright holder for this preprint (which was not certified by peer review) is the author/funder, who has granted bioRxiv a license to display the preprint in perpetuity. It is made available under aCC-BY-NC-ND 4.0 International license.

Table 1 Summary of data-generating models for simulations

\begin{tabular}{llll}
\hline Simulation model & Description & Non-zero parameters & $\begin{array}{l}\text { Number of non-zero parame- } \\
\text { ters }\end{array}$ \\
\hline biallelic_add & Additive only with two alleles & $\mu, \beta_{\text {inbred }},\left(a_{1}, a_{2}\right)$ & 4 \\
\hline rnorm_add & Additive only with $a \sim \mathrm{N}\left(0, \tau_{a}^{2}\right)$ & $\mu, \beta_{\text {inbred }},\left(a_{1}, \ldots, a_{8}\right)$ & 10 \\
\hline complex & Includes all strain-level effects & $\mu, \beta_{\text {inbred }}$, & 82 \\
& additive: $a_{i} \sim \mathrm{N}\left(0, \tau_{a}^{2}\right)$ & $\left(a_{1}, \ldots, a_{8}\right)$, & \\
& inbred: $b_{i} \sim \mathrm{N}\left(0, \tau_{b}^{2}\right)$ & $\left(b_{1}, \ldots, b_{8}\right)$, \\
& maternal: $m_{i} \sim \mathrm{N}\left(0, \tau_{m}^{2}\right)$ & $\left(m_{1}, \ldots, m_{8}\right)$, \\
& symmetric epistasis: $v_{i j} \sim \mathrm{N}\left(0, \tau_{v}^{2}\right)$ & $\left(v_{1}, \ldots, v_{28}\right)$, \\
& asymmetric epistasis: $w_{i j} \sim \mathrm{N}\left(0, \tau_{w}^{2}\right)$ & $\left(w_{1}, \ldots, w_{28}\right)$ \\
\hline
\end{tabular}

3. complex: The additive, inbred, maternal, and both epistatic effects are all simulated such that $a_{j}, b_{j}, m_{j}, v_{j k}, w_{j k} \sim$ $\mathrm{N}(0,1)$. The proportional of total variance is evenly split between the five strain-level effect types.

Table 1 describes these three diallel data-generating models, including the type and number of non-zero effects.

\section{Availability of data and software}

All analyses were conducted in the statistical programming language R (?). The R package DIDACT (Diallel Informed Decision theoretic Approach for Crosses Tool), available on GitHub at https://github.com/gkeele/DIDACT, can estimate strain-level effects from diallel data using a Bayesian hierarchical model, and then perform the posterior utility analysis. The R package BayesDiallel can alternatively be used to estimate the strain-level effects, and potentially used as inputs to DIDACT.

DIDACT includes three diallel data sets, each with a number of phenotypes, of the CC founders (Churchill et al. 2004; Collaborative Cross Consortium 2012; Srivastava et al. 2017), described in great detail in Lenarcic et al. (2012). Specifically, results shown here are from a hemoglobin trait measured in 626 mice. The $\mathrm{CC}$ founders represent the following inbred strains of mouse (abbreviated names in parentheses): A/J (AJ), C57BL/6J (B6), 129S1/SvImJ (129), NOD/ShiLtJ (NOD), NZO/H1LtJ (NZO), CAST/EiJ (CAST), PWK/PhJ (PWK), and WSB/EiJ (WSB).

An additional diallel data set of the $C C$ founders of response to Influenza A virus (IAV) infection phenotypes is used here and included in the DIDACT package, with the original data available at https://github.com/mauriziopaul/flu-diallel. In previous work (Maurizio et al. 2018), we investigated strain-level effects in day four post-infection (D4 p.i. ) body weight loss percentage in a diallel of the CC founders. The phenotype of interest is a response to infection, in which three infected animals were compared to a single mock-infected animals. Occasionally three infected animals were not observed at later time points, which was accounted for through a multiple imputation procedure that imputed unobserved animals from the posterior predictive distributions of the BayesDiallel model (Lenarcic et al. 2012). Here only a singly imputed data set of 131 outcomes is used, as this example is a proof of principle for DIDACT, and not a rigorous investigation of strain-level effects.

\section{Results}

We provide results from simulations and example analyses from diallel data of the CC founders to demonstrate the decision theoretic procedure used in the DIDACT approach. The use of QTL mapping power as a utility function depends on assumptions about the effect of a single putative QTL in a bi-parental cross (described in Table S1) given strain-level effects estimated from diallel data based on the parameterization described in Eq 7. This assumption is most straightforward in the case of a largely Mendelian phenotype, in which a single locus modulates the variation observed in a relatively deterministic manner, and as such, the QTL effect $\theta$ can draw from the strain-level effect $\phi$ wholly. When the genetic architecture of the phenotype is complex, as is likely with many traits, the use of predicted phenotype contrasts requires less assumptions, as the nominal interpretation of power is no longer valid.

\section{Simulations}

100 simulations per 50 levels of strain-level effect sizes (evenly spaced between 0.01 and 0.99 ) for three different model types were used to evaluate strain-level effect estimation accuracy, and more importantly, DIDACT's utility estimation. For effect estimation accuracy, the averaged mean squared error (MSE) of the additive strain-level effects $(\boldsymbol{a})$ was used, calculated as:

$$
\operatorname{MSE}_{\boldsymbol{a}}=\sum_{i=1}^{J}\left(\widehat{a}_{i}-a_{i}\right)^{2}
$$

where $J$ is the number of founders ( 8 in the CC) and $\widehat{a}$ is the posterior mean of $a$. For utility estimation accuracy, the rank correlation of posterior contrasts was used.

DIDACT can effectively estimate strain-level effects, but more importantly, is consistently accurate at ranking posterior utilities (Figure 3). The simulations show that DIDACT can both accurately estimate the additive strain-level effects when those are the only non-zero effects in the data (Figures $\mathbf{3} \mathbf{A}$ and $\mathbf{B}$ ). When many non-zero and non-additive strain-level effects are present, the accuracy of the effect estimation is reduced (Figure $3 \mathrm{C}$ ). Despite this reduction in effect estimation accuracy for the BayesDiallel model in a complex setting, DIDACT still estimates and ranks utilities effectively (Figure $3 \mathbf{F}$ ) in comparison to the simpler additive settings (Figures $\mathbf{3} \mathbf{D}$ and $\mathbf{E}$ ). 
bioRxiv preprint doi: https://doi.org/10.1101/489682; this version posted December 7, 2018. The copyright holder for this preprint (which was not certified by peer review) is the author/funder, who has granted bioRxiv a license to display the preprint in perpetuity. It is made available under aCC-BY-NC-ND 4.0 International license.
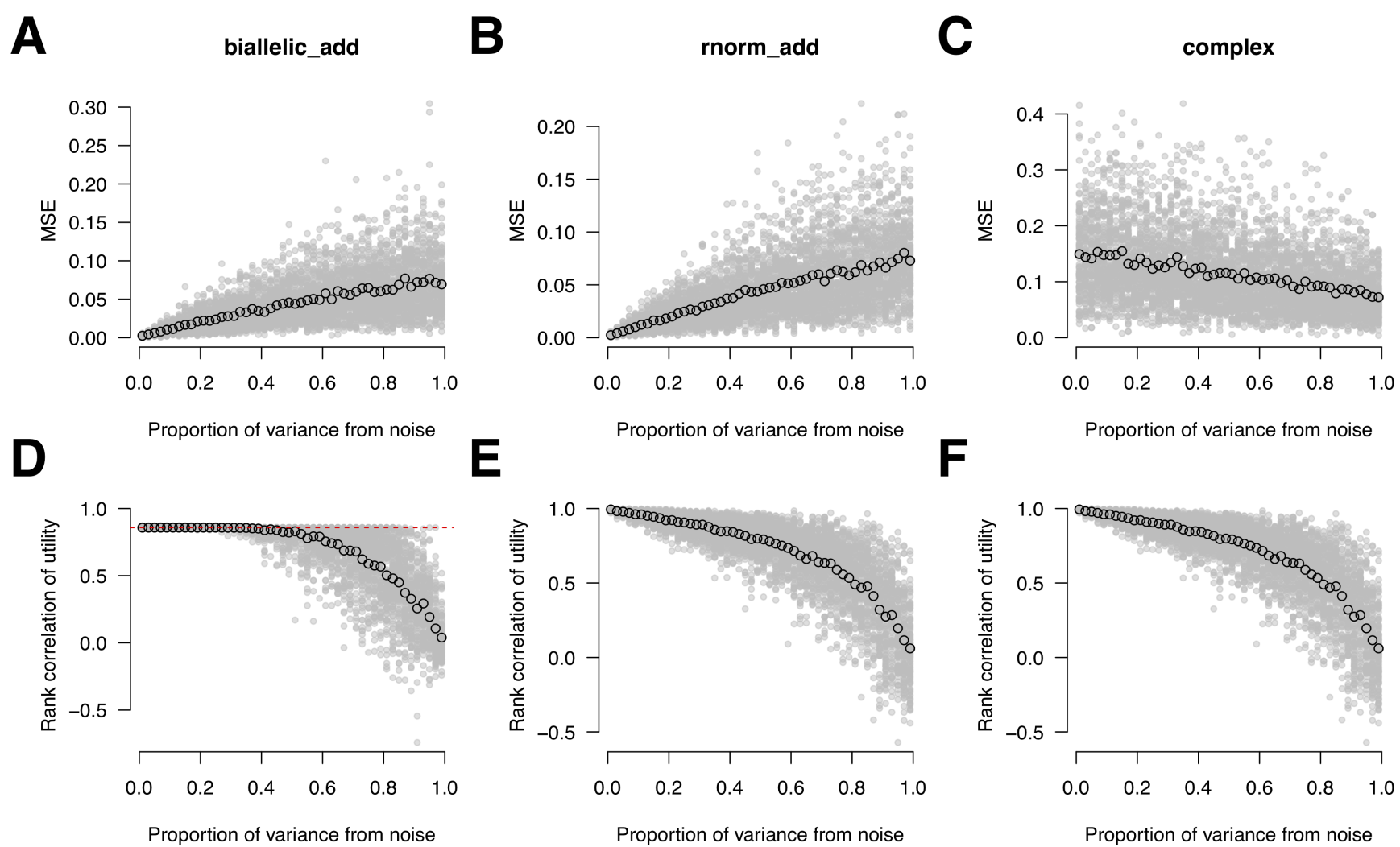

Figure 3 The BayesDiallel model's estimates of the additive strain-level effects improve with decreasing proportion noise variance (A-B). When the underlying strain-level effects are complex, the BayesDiallel model struggles to accurately estimate the additive effects, even with decreasing variation due to noise (C). DIDACT's ranking of posterior contrasts for simulated diallel crosses of varying complexity improves as proportion noise variation decreases (D-F), suggesting that even though effect estimation is challenging in the presence of complex strain effects, DIDACT's posterior utility is robust to this complexity. 100 diallel crosses were simulated per level of variance explained (50 values evenly spaced from 0 to 1 ) per model type. biallelic_add is a Mendelian QTL with two alleles distributed evenly amongst the founder strains. rnorm_add is also an additive-only model but with $a \sim \mathrm{N}\left(0, \tau^{2}\right)$, where $\tau^{2}$ is one minus the proportion of variance determined by noise. complex has a complex set of non-zero strain-level effects for additive, inbred, maternal, and both symmetric and asymmetric epistatis, each contributing equally to the overall variance. Gray dots represent single simulations, and black circles the mean over the 100 simulations. The red dashed line in D marks the max rank correlation $(<1)$ for biallelic_add due to the true utilities at a Mendelian locus containing ties whereas the estimated ones will be continuous.

\section{Mendelian phenotype}

To demonstrate a straightforward application of DIDACT to a phenotype largely driven by a single locus, we use resistance to IAV infection and the $M x 1$ gene. As described in greater detail in the Methods, the phenotype of interest is day four postinfection (D4 p.i. ) body weight loss percentage in a diallel of the CC founders.

Mx1 is a critical host-resistance factor in mice. It has previously been shown that Mx1 largely drives IAV-resistance in the CC founders, and has three major functional classes corresponding to the three subspecies of Mus musculus: domesticus (hereafter dom; CC founders with dom allele are AJ, B6, 129, NOD, and WSB), castaneus (cast; CAST), and musculus (mus; PWK and NZO) (Ferris et al. 2013). The dom allele of $M x 1$ was found to be functionally null and those individuals susceptible to IAV infection, whereas mus and cast confer degrees of resistance.

Though IAV-resistance is largely driven by $M x 1$, the genetic variation at the gene in the diallel of CC founders is more complicated than a bi-allelic locus. Instead, $M x 1$ has multiple func- tional alleles, mus and cast contrasting the null allele dom. mus has a dominant mode of action, conferring approximately the same level of resistance to IAV in dom/mus individuals as in mus / mus, whereas cast is additive with cast/dom being intermediate between dom / dom (low resistance) and either mus carriers or cast / cast (highest resistance).

The increased IAV-resistance of mus and cast is apparent in the raw data as darker horizontal and vertical bands for NZO and PWK, and to a lesser extent for CAST (Figure 4A). The raw data also suggests that WSB may posses additional modifiers of resistance with some hybrids displaying high resistance for individuals with homozygous dom. The resistance conferred by variation at $M x 1$ is further confirmed in the strain-level effects estimated through BayesDiallel, highlighted in Figure 4B.

DIDACT favors crosses with segregating $M x 1$ variants. For a Mendelian phenotype, DIDACT should prefer crosses that maintain segregating alleles at the locus, in this case $M x 1$. In particular, it should prioritize crosses that match dom with mus or cast. Crosses with no segregating functional variants of $M x 1$ will 

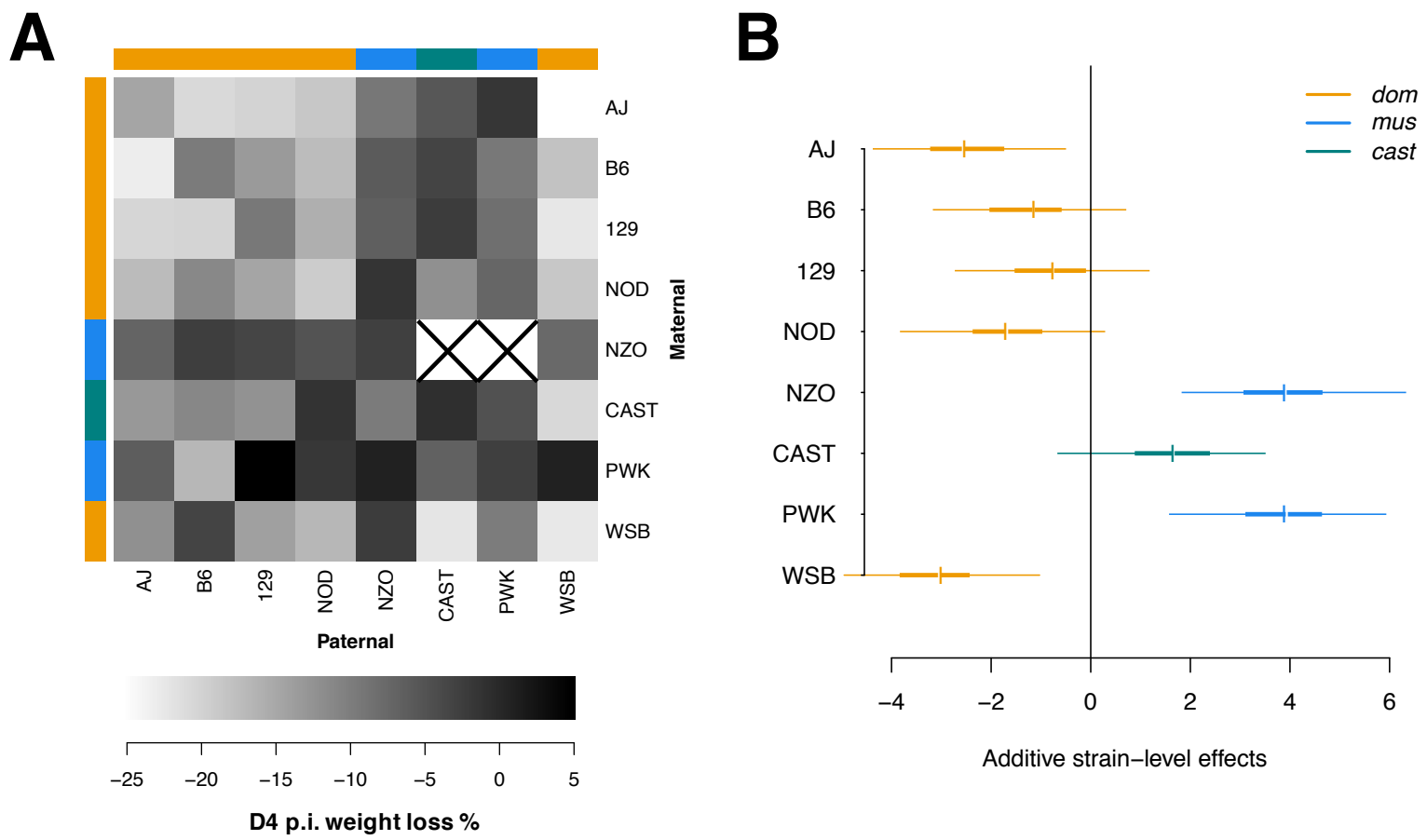

Figure $4 M x 1$ is as a driver of IAV-resistance. This is manifested in the raw data of D4 p.i. body weight loss \% in a diallel of the CC founders and their hybrids (381 mice) as relatively dark vertical and horizontal bands at the NZO, CAST, and PWK founders, representing low percent weight loss. (A). Squares with a black "X" represent crosses that produce no viable offspring $\left(\mathrm{NZO} \times \mathrm{PWK}^{\top}\right.$ and $\mathrm{NZO} \propto \times$ CAST $\sigma^{\top}$ ). The subspecies allele at $M x 1$ for each CC founder strain is indicated by colored bars below and to the right of the phenotype grid, with orange representing dom, blue mus, and green cast. The mus and cast alleles are functional, whereas the dom is functionally null. Moreover, the mus allele is dominant in comparison to cast. The strain-level additive effects estimated from the BayesDiallel model correspond to the subspecies lineages at the Mx1 locus, with mus (NZO and PWK) conferring more resistance than cast (CAST) (B). The highest posterior density (HPD) intervals from the BayesDiallel strain-level additive effects are shown with $95 \%$ HPD as thin lines and $50 \%$ HPD as thick lines. The posterior means and medians are indicated as colored and white ticks, respectively. The effects closely match those estimated in Maurizio et al. (2018), which used a more complex BayesDiallel model, and summarized over multiply imputed data sets.

have greatly reduced phenotypic variation, and ultimately not be able to detect the largely Mendelian locus.

As expected, DIDACT largely estimates high QTL mapping power for crosses that possess multiple segregating $M x 1$ alleles, either dom with mus or cast. Mean posterior power from DIDACT for F2 experiments are shown in Figure 5A and for BC experiments in Figure 5B. For F2, PWK $\times$ WSB and NZO $\times$ WSB are ranked $1^{\text {st }}$ and $2^{\text {nd }}$ in posterior power, respectively. CAST $\times$ PWK is ranked $3^{\text {rd }}$, which matches the expectations that mus $\times$ dom are preferable to cast $\times$ dom. WSB is likely preferred as the dom carrier because of its relatively unique phenotype. In general, other crosses that maintain multiple segregating alleles of $M x 1$ are preferred in comparison to crosses that fix the variability at the locus.

For BC, similar patterns are observed. Though subtle, DIDACT generally favors $\mathrm{BC}$ for crosses of dom and mus in which the backcrossed parent strain (A) has dom, thus reflecting the dominance of mus. In the case of a completely dominant QTL, there would be no power to map the QTL in a cross of $A \times B$, given that $\mathrm{A}$ is the backcrossed parent and possesses the dominant allele. For example with $M x 1$, WSB $(\mathrm{A}) \times \mathrm{PWK}(\mathrm{B})$ is ranked $1^{\text {st }}$ whereas $\mathrm{PWK}(\mathrm{A}) \times \mathrm{WSB}(\mathrm{B})$ is $5^{\text {th }}$. Though not perfect, reflecting the complexities of real data and a phenotype with potentially additional genetic modifiers, DIDACT largely favors crosses of $\operatorname{dom}(\mathrm{A}) \times$ mus $(\mathrm{B})$, given a specific pairing of strains.

As with the F2, DIDACT prefers crosses of WSB (dom) with strains that possess mus or cast, likely reflecting strain-level WSB effects that are independent of Mx1 (Maurizio et al. 2018). It is also important to reiterate that this analysis of $M x 1$ represents a single imputation of multiply imputed data, representing an additional source of noise.

\section{Complex trait: calculated hemoglobin}

Many phenotypes are the not Mendelian, but rather the product of potentially many loci across the genome and complicated genetic architectures. Such traits violate the assumption of attributing the strain-level effects to a single QTL. DIDACT can still be used to analyzed these complex traits. Though the power utility function is still meaningful in selecting crosses of phenotypically distinct strains, the single QTL assumption can be relaxed by using the phenotypic contrasts directly as the utility function. Here, contrasts are used for calculated hemoglobin (cHGB), which is likely not Mendelian.

As reported in Lenarcic et al. (2012), blood phenotypes were measured on 626 mice from a diallel of the CC founders, which included cHGB ( $\mathrm{g} / \mathrm{dl})$, an estimate of the quantity of hemoglobin in the blood (Figure 6A). The raw data do not suggest obvious 
A
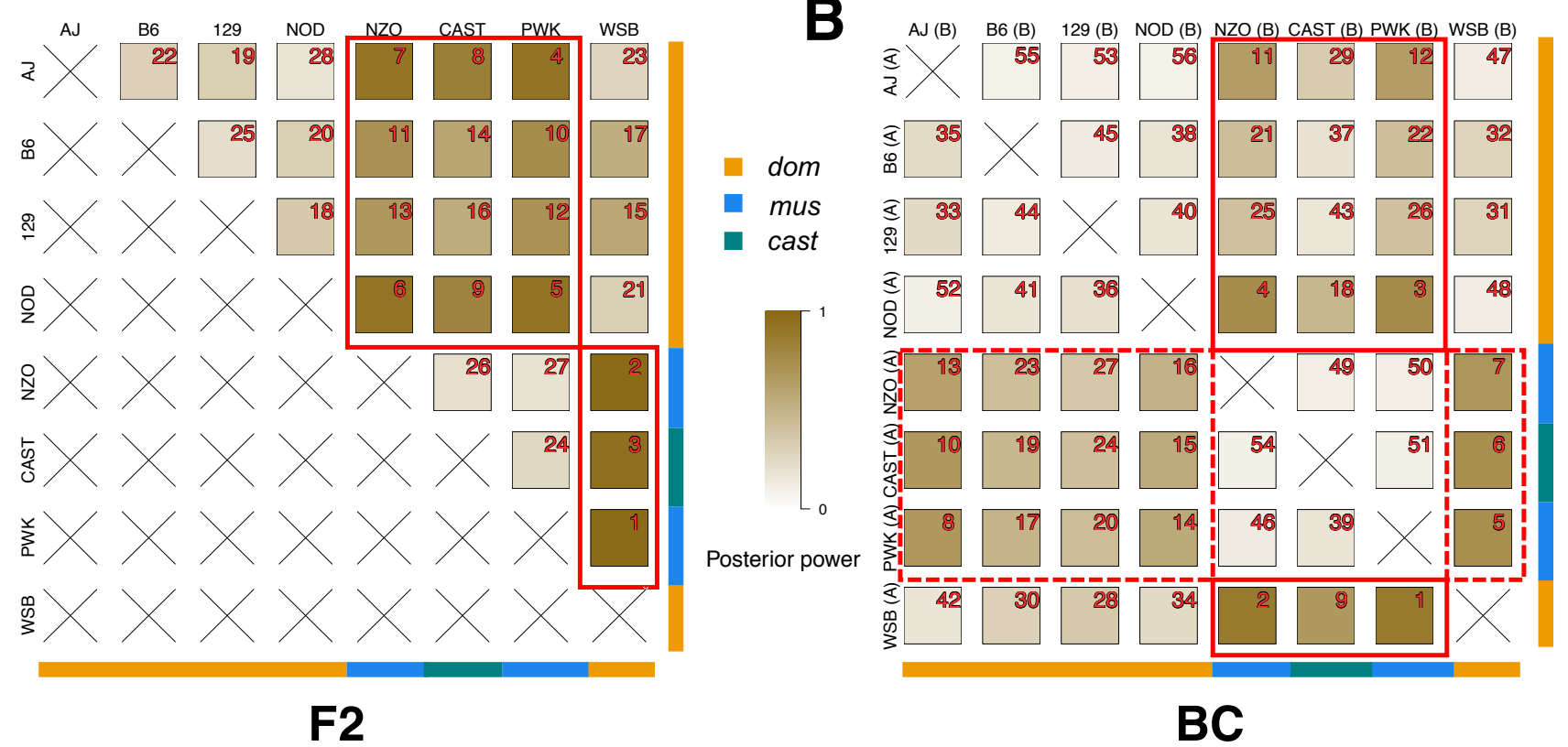

Figure 5 For IAV resistance measured as D4 p.i. weight loss \%, DIDACT ranks F2 and BC experiments that maintain genetic variability at $M \times 1$ higher. Posterior mean utility as power to map a single QTL in 60 mice, for the 28 possible F2 crosses (A) and the 56 possible BC (B) of the $\mathrm{CC}$ founder strains. The red number in the top right corner of a cross square represents the posterior power rank for a given cross type (F2 or BC). Subspecies allele at $M x 1$ is indicated by colored bars below and to the right of the grids, with orange representing dom, blue mus, and green cast. For F2, the solid red squares enclose crosses that pair functional alleles (mus and cast) of Mx1 with dom. For $\mathrm{BC}$, the solid red squares enclose crosses that pair strains with functional alleles with strains with dom and the backcrossed parent has dom. The red dashed squares also pair functional with nonfunctional alleles of $M \times 1$, but the backcrossed parent has the functional allele instead. Backcrossing strains with mus are expected to have reduced power due to the dominance of the allele.

additive strain-level effects as seen with D4 p.i. weight loss \% (Figure 6A), though great phenotypic variability is observed, and patterns that reflect complex strain-level effects. CAST individuals have a notably lower trait-value. Additionally, PWK hybrids with AJ, B6, and 129 have unusually high trait-values.

These patterns are reflected in the BayesDiallel estimates of non-zero strain-level effects (Figure 6B). CAST has a nonzero negative inbred effect. For symmetric epistatis, PWK has positive non-zero effects with $\mathrm{AJ}$ and 129 , and a suggestive positive effect with B6. CAST also has a non-zero positive effect with AJ, which is also consistent with the raw data. Stable effects are based on observations across multiple crosses and replicates of each pairing, thus likely representing heritable factors.

The posterior contrasts for possible $\mathrm{F} 2$ and $\mathrm{BC}$ mapping crosses are shown in Figures 6C and 6D, respectively. DIDACT's ranking of contrasts largely prefers crosses with CAST, corresponding to its non-zero strain-level effects. Notably For $\mathrm{BC}$, the strongly negative CAST inbred effect is manifested in DIDACT estimating higher posterior power for BC in which CAST is the backcrossed parent (A). If only CAST hybrids are observed, the mapping cross will not produce as much phenotypic variation, which stems from the CAST homozygotes in this trait. DIDACT can dynamically take into account complex strain-level effects, and rank crosses accordingly.

\section{Additional DIDACT summaries}

DIDACT can provide more detailed descriptions of the predicted bi-parental crosses than shown in Figures 5 and 6 . At its core,
DIDACT is an extension of the BayesDiallel model, in which the strain-level effects from the Bayesian hierarchical model are propagated to predetermined utility functions, and as such, posterior intervals can be produced in addition to the point estimates for any function of the model parameters.

Three potential F2 crosses were selected from the full panel from cHGB (Figure 6C), and are presented in Figure S2. Additional summaries include a histogram of the posterior distribution of the utility function, in this case contrasts, the median utility represented as a vertical dashed line on the histogram, predicted phenotypes for each QTL genotype (based on a single QTL assumption) as bar plots, and the phenotypic variation attributable to the strain-level effects as a pie chart, all overlayed on the square with posterior mean determining the background color. CAST $\times$ WSB has higher mean posterior contrast compared to NZO $\times$ WSB and AJ $\times$ B6. This is reflected in phenotype predictions that vary more greatly (bar plots) and an estimated higher proportion of phenotypic variance explained by the strain-level effects (63\%).

\section{Parent-of-origin effects and $R B C^{P O}$}

There is not currently a satisfactory approach and solution for parameterizing QTL effects that contain a POE mode of action, such as exists for additivity and dominance as described in Eq $\mathbf{4}$ and $\mathbf{5}$ as well as in Tables S1 and S2, which ultimately limits the ability of DIDACT to make power calculations for $\mathrm{RBC}^{\mathrm{PO}}$ as described in Figure 1C. However, it is possible for DIDACT to characterize the utility in terms of predicted BC, but 

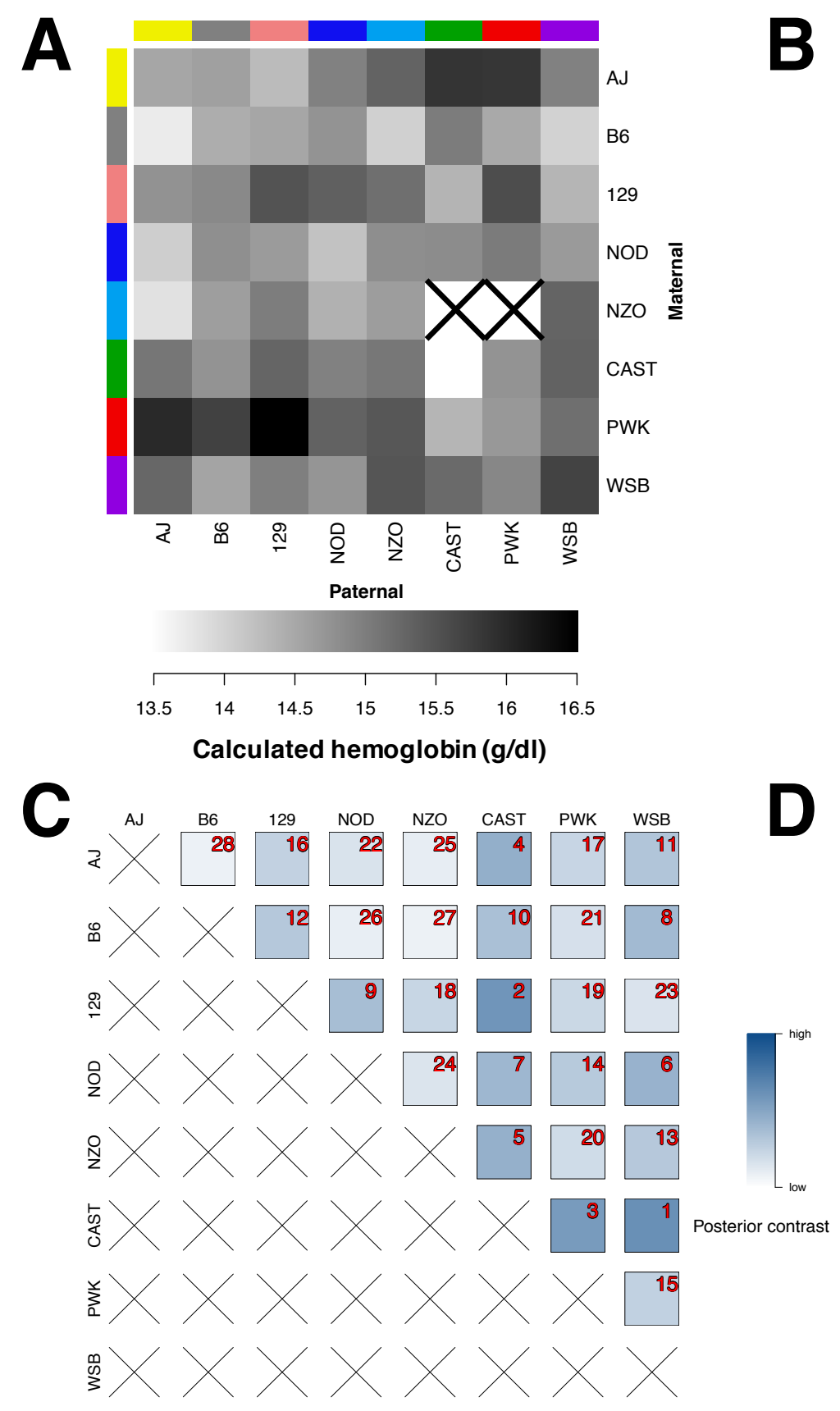

F2
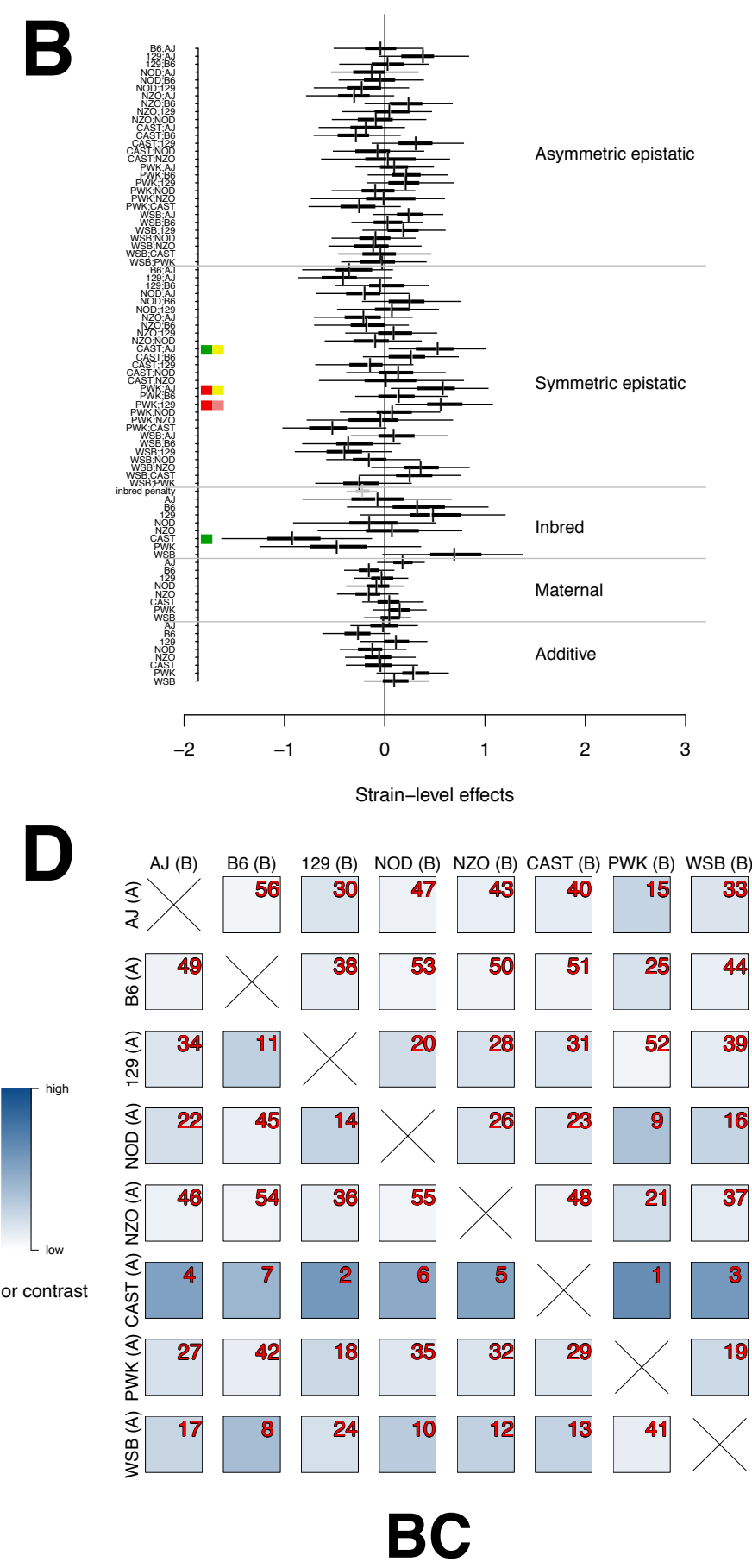

Figure 6 DIDACT can analyze complext traits, such as calculated hemoglobin (CHGB) in a diallel of the CC founder strains of 626 mice. The mean raw data for CHGB suggests inbred effect for CAST (A). A black "X" represents crosses that did not produce viable offspring. BayeDiallel estimates a number of non-zero strain-level effects (B). Effects are represented as HPD intervals, with $95 \%$ and $50 \%$ as thick and thin lines, respectively, and colored and white ticks representing posterior means and medians, respectively. The gray bar represents the the overall inbred parameter. A Green square along the $y$-axis highlights a non-zero inbred CAST effect. Non-zero symmetric epistatic effects are similarly emphasized for PWK $\times$ AJ, PWK $\times 129$, and CAST $\times$ AJ. DIDACT posterior contrasts for F2 (C) and BC (D) experiments rank crosses with CAST highly. 
with the maternal and paternal identities fixed as in the $\mathrm{RBC}^{\mathrm{PO}}$. Though the power calculation will not correspond to the design specified in Figure 1C, in which three genetic states are observed in comparison to two for BC, differences in QTL mapping power for $\mathrm{BC}$ that are equivalent except for the maternal and paternal statuses of the backcrossed parental strain and F1 are potentially interesting, shown in Figure S3 for cHGB.

$\mathrm{RBC}^{\mathrm{PO}}$ that have markedly different posterior contrasts can identify pairings of strains with interesting combinations strainlevel maternal effects in Figure 6B. The BayesDiallel model estimates several suggestive strain-level maternal effects, primarily $\mathrm{AJ}$ and $\mathrm{B} 6$, suggesting that different $\mathrm{RBC}^{\mathrm{PO}}$ may have varying posterior contrasts. For example $\mathrm{B} 6(\mathrm{~A} \rho) \times \mathrm{PWK}\left(\mathrm{B}_{\odot}{ }^{7}\right)$ is ranked $50^{\text {th }}$ compared to $13^{\text {th }}$ for B6 $\left(\mathrm{A}_{\sigma^{x}}\right) \times$ PWK (Bo) (Figure S3). More work is needed to formally extend analytical expressions of QTL mapping power to $\mathrm{RBC}^{\mathrm{PO}}$, but posterior utility can still be used to highlight interesting crosses based on the diallel data.

\section{Discussion}

DIDACT represents an approach to selecting experiments using the information contained within diallel crosses. In this approach, the diallel cross is used as pilot data to characterize strain-level genetic effects within a Bayesian hierarchical model, which are then applied to utility functions for the purposes of identifying promising bi-parental crosses for mapping QTL. Herein, we define utility to be QTL mapping power, though other functions could be used, such as phenotype contrasts, so long as the strain-level effects are their inputs.

\section{Violation of assumptions connecting strain-level effect to QTL effect}

DIDACT requires assumptions to connect the strain-level diallel effects to experimental design-relevant utility functions. How easily these spaces are connected will vary with the complexity of the phenotype. DIDACT performs well in a mostly Mendelian phenotype in which the strain-level effects can be correctly attributed to a single putative QTL. However, phenotypes that are highly heritable can be modulated through many loci, often with few to none exhibiting large effects, height in humans being a clear example (Wood et al. 2014). In the vast majority of complex traits, the assumption of a single QTL absorbing all or most of the strain-level effects is wildly optimistic. However, we posit that though the assumption is unlikely, its use as a utility function can still produce a useful analysis of potential bi-parental crosses.

The power utility function used in DIDACT favors QTL that explain a large proportion of the variability in the phenotype. In fact, the power function corresponds closely to the variability explained by the putative QTL, which will relate to the variability explained by strain identity in the diallel in the context of complex but heritable phenotypes. Alternatively, the phenotype contrasts could be used as the utility function instead. Though the interpretation of the posterior utility as an accurate power is unrealistic, it will still select pairings that are phenotypically distinct, which is a common criterion for selecting crosses. And, it will do so in a highly principled approach that intuitively accounts for uncertainty.

\section{Genetic similarity of strains}

DIDACT, in its current form, does not make use of any information regarding the similarity of the parental strains included in the diallel cross, which could further inform how appealing an experimental cross is for fine-mapping detected QTL. The reduced complexity cross (RCC) is a developing approach in systems genetics (Williams and Williams 2017) in which strains that are phenotypically divergent but genetically similar are crossed, such as C57BL/6J and C57BL/6N sub-strains (Khisti et al. 2006; Mulligan et al. 2008; Kumar et al. 2013; Simon et al. 2013; Kirkpatrick and Bryant 2014). RCC provide a powerful tool for fine-mapping causal variants because the genetic variability between strains is greatly reduced.

There are a number of ways that DIDACT could be modified to incorporate genetic similarity information, probably most simply through the utility function. The utility function could be expanded to flexibly weight potential experimental crosses by the genetic similarity, resulting in posterior utilities that are informed by both phenotype and genetic similarity. We believe this highlights the potential of DIDACT, and its underlying concept in general, to be flexible to the context of the experimental system, at the hierarchical model, but particularly at the utility function.

\section{Extension to multiparental populations}

The DIDACT analyses presented here are from diallel crosses of the CC founders, which naturally poses the question of designing experiments of the $\mathrm{CC}$ strains based on their related diallel data. The CC population represents a multiparental population (MPP), in which each individual is descended from all of the founder strains. Extending DIDACT to experiments of an MPP RI panel is challenging because the analytical power calculations (Sen et al. 2005, 2007) are based on bi-parental populations with two founder alleles. Though these could be generalized to populations with multiple alleles, the CC strains also differ from traditional mapping crosses in that the recombination events that randomize segments of the genome to allow for QTL mapping have already occurred in the breeding scheme that derived the inbred strains.

Maybe a more natural approach to extending DIDACT to an MPP RI panel would be to consider the MPP RI panel as a large sparse diallel, with off-diagonal cells representing the F1 hybrids, in the case of the CC, these would be CC-RIX (Bogue et al. 2015). DIDACT could then be adapted to select potentially interesting but unobserved CC-RIX based on the CC-specific strain-level effects. Effectively adapting DIDACT for design of MPP experiments is an area of interest for future research.

\section{Summary}

DIDACT is a novel approach to using prior collected diallel data from a panel of inbred strains to inform the selection of potential downstream experiments according to a user-specified utility function, in our case, power to map QTL in bi-parental cross experiments, consisting of $\mathrm{F} 2, \mathrm{BC}$, and $\mathrm{RBC}^{\mathrm{PO}}$. The core of this approach is to propagate the uncertainty present in the Bayesian hierarchical model through to the utility functions, which can be customized to the needs and constraints of the system at hand.

As proof of principle, we evaluated DIDACT in a phenotype known to be Mendelian: resistance to IAV-infection, which is largely modulated by the $M x 1$ gene with a null (susceptible) and two non-null (resistant) alleles. DIDACT largely evaluated bi-parental crosses of null with non-null $M x 1$ strains as having higher posterior power to map the QTL. For the non-Mendelian calculated hemoglobin, DIDACT favors crosses that pair strains with contrasting phenotypes. Though the posterior power as utility, in the sense of its nominal interpretation as power, is 
highly optimistic, still provides a reasonable metric for comparing potential experiments, given the available pilot data. This approach has many potential applications, in terms of both the utility functions that are being evaluated and the model organism systems, many which have sparse diallel data available in the form of strain surveys. DIDACT represents a philosophical advancement in terms of good experimental design and efficient use of available resources.

\section{Acknowledgments}

We thank Amy Herring, now at Duke University, for her thoughts and suggestions on this paper, which began as a group project in her Bayesian statistics course at UNC. We also thank Alan Lenarcic for his expertise on Bayesian modeling of diallel crosses.

This work was primarily supported by the National Institute of General Medical Sciences under awards R01-GM104125 and R35-GM127000 (to W.V.).

\section{Literature Cited}

Bahari, M., M. Y. Rafii, G. B. Saleh, and M. A. Latif, 2012 Combining Ability Analysis in Complete Diallel Cross of Watermelon ( Citrullus lanatus (Thunb.) Matsum. \& Nakai). The Scientific World Journal 2012: 1-6.

Bérénos, C., P. A. Ellis, J. G. Pilkington, and J. M. Pemberton, 2014 Estimating quantitative genetic parameters in wild populations: a comparison of pedigree and genomic approaches. Molecular ecology 23: 3434-51.

Bogue, M. A., G. A. Churchill, and E. J. Chesler, 2015 Collaborative Cross and Diversity Outbred data resources in the Mouse Phenome Database. Mammalian Genome 26: 511-520.

Broman, K. W., 2001 Review of statistical methods for QTL mapping in experimental crosses. Lab animal 30: 44-52.

Casares, P., M. C. Carracedo, R. Piñeiro, E. S. Miguel, and L. Garcia-Florez, 1992 Genetic basis for female receptivity in Drosophila melanogaster: a diallel study. Heredity 69: 400405.

Churchill, G. A., D. C. Airey, H. Allayee, J. M. Angel, A. D. Attie, et al., 2004 The Collaborative Cross, a community resource for the genetic analysis of complex traits. Nature Genetics 36: 1133-1137.

Collaborative Cross Consortium, 2012 The genome architecture of the Collaborative Cross mouse genetic reference population. Genetics 190: 389-401.

Connolly, S. and E. a. Heron, 2015 Review of statistical methodologies for the detection of parent-of-origin effects in family trio genome-wide association data with binary disease traits. Briefings in Bioinformatics 16: 429-448.

Crowley, J. J., Y. Kim, A. B. Lenarcic, C. R. Quackenbush, C. J. Barrick, et al., 2014 Genetics of adverse reactions to haloperidol in a mouse diallel: A drug-placebo experiment and Bayesian causal analysis. Genetics 196: 321-347.

Dos Santos, E. A., A.-A. F. de Almeida, D. Ahnert, M. C. d. S. Branco, R. R. Valle, et al., 2016 Diallel Analysis and Growth Parameters as Selection Tools for Drought Tolerance in Young Theobroma cacao Plants. PloS one 11: e0160647.

Dupuis, J. and D. Siegmund, 1999 Statistical methods for mapping quantitative trait loci from a dense set of markers. Genetics 151: 373-86.

Fairfull, R. W., R. S. Gowe, and J. A. B. Emsley, 1983 Diallel cross of six longterm selected leghorn strains with emphasis on heterosis and reciprocal effects. British Poultry Science 24: 133-158.

Ferris, M. T., D. L. Aylor, D. Bottomly, A. C. Whitmore, L. D. Aicher, et al., 2013 Modeling Host Genetic Regulation of Influenza Pathogenesis in the Collaborative Cross. PLoS Pathogens 9: e1003196.

Ghareeb Zeinab, E. and A. Helal, 2014 Diallel analysis and separation of genetic variance components in eight faba bean genotypes. Annals of Agricultural Sciences 59: 147-154.

Gilbert, N. E. G., 1958 Diallel cross in plant breeding. Heredity 12: 477-492.

Gonzalez, P. N., M. Pavlicev, P. Mitteroecker, F. Pardo-Manuel de Villena, R. A. Spritz, et al., 2016 Genetic structure of phenotypic robustness in the collaborative cross mouse diallel panel. Journal of Evolutionary Biology 29: 1737-1751.

Gonzalo, M., T. J. Vyn, J. B. Holland, and L. M. McIntyre, 2007 Mapping reciprocal effects and interactions with plant density stress in Zea mays L. Heredity 99: 14-30.

Greenberg, A. J., S. R. Hackett, L. G. Harshman, and A. G. Clark, 2010 A Hierarchical Bayesian Model for a Novel Sparse Partial Diallel Crossing Design. Genetics 185: 361-373.

Griffing, B., 1956 Concept of General and Specific Combining Ability in Relation to Diallel Crossing Systems. Australian Journal of Biological Sciences 9: 463.

Haley, C. S. and S. A. Knott, 1992 A simple regression method for mapping quantitative trait loci in line crosses using flanking markers. Heredity 69: 315-24.

Harper, K. M., E. Tunc-Ozcan, E. N. Graf, L. B. K. Herzing, and E. E. Redei, 2014 Intergenerational and parent of origin effects of maternal calorie restriction on Igf2 expression in the adult rat hippocampus. Psychoneuroendocrinology 45: 187-91.

Ivy, T. M., 2007 Good genes, genetic compatibility and the evolution of polyandry: use of the diallel cross to address competing hypotheses. Journal of Evolutionary Biology 20: 479-487.

Jannink, J.-L. and X.-L. Wu, 2003 Estimating allelic number and identity in state of QTLs in interconnected families. Genetical research 81: 133-44.

Khisti, R. T., J. Wolstenholme, K. L. Shelton, and M. F. Miles, 2006 Characterization of the ethanol-deprivation effect in substrains of C57BL/ 6 mice. Alcohol (Fayetteville, N.Y.) 40: 11926.

Kirkpatrick, S. L. and C. D. Bryant, 2014 Behavioral architecture of opioid reward and aversion in C57BL/ 6 substrains. Frontiers in behavioral neuroscience 8: 450.

Kumar, V., K. Kim, C. Joseph, S. Kourrich, S.-H. Yoo, et al., 2013 C57BL/6N mutation in cytoplasmic FMRP interacting protein 2 regulates cocaine response. Science (New York, N.Y.) 342: 1508-12.

Lander, E. S. and D. Botstein, 1989 Mapping mendelian factors underlying quantitative traits using RFLP linkage maps. Genetics 121: 185-99.

Lawson, H. A., J. M. Cheverud, and J. B. Wolf, 2013 Genomic imprinting and parent-of-origin effects on complex traits. Nature reviews. Genetics 14: 609-17.

Lenarcic, A. B., K. L. Svenson, G. A. Churchill, and W. Valdar, 2012 A general Bayesian approach to analyzing diallel crosses of inbred strains. Genetics 190: 413-35.

Li, L., C. Sun, Y. Chen, Z. Dai, Z. Qu, et al., 2013 QTL mapping for combining ability in different population-based NCII designs: A simulation study. Journal of Genetics 92: 529-543.

Liu, Y. and Z.-B. B. Zeng, 2000 A general mixture model approach for mapping quantitative trait loci from diverse cross 
designs involving multiple inbred lines. Genetical research 75: 345-55.

Lynch, M. and B. Walsh, 1998 Genetics and Analysis of Quantitative Traits. Sinauer Associates, Sunderland, MA.

Maurizio, P. L., M. T. Ferris, G. R. Keele, D. R. Miller, G. D. Shaw, et al., 2018 Bayesian Diallel Analysis Reveals Mx1 -Dependent and Mx1 -Independent Effects on Response to Influenza A Virus in Mice. G3 (Bethesda, Md.) 8: 427-445.

Mulligan, M. K., I. Ponomarev, S. L. Boehm, J. A. Owen, P. S. Levin, et al., 2008 Alcohol trait and transcriptional genomic analysis of C57BL/ 6 substrains. Genes, brain, and behavior 7: 677-89.

Nystrand, M., D. K. Dowling, and L. W. Simmons, 2011 Complex Genotype by Environment interactions and changing genetic architectures across thermal environments in the Australian field cricket, Teleogryllus oceanicus. BMC Evolutionary Biology 11: 222.

Parsons, P. A., 1964 A diallel cross for mating speeds in Drosophila melanogaster. Genetica 35: 141-151.

Raiffa, H. and R. Schlaifer, 2000 Applied Statistical Decision Theory. John Wiley \& Sons, INC., New York, NY, wiley clas edition.

Rantala, M. J. and D. A. Roff, 2006 Analysis of the importance of genotypic variation, metabolic rate, morphology, sex and development time on immune function in the cricket, Gryllus firmus. Journal of Evolutionary Biology 19: 834-843.

Rebaï, A. and B. Goffinet, 1993 Power of tests for QTL detection using replicated progenies derived from a diallel cross. TAG. Theoretical and applied genetics. Theoretische und angewandte Genetik 86: 1014-22.

Rebaï, A. and B. Goffinet, 2000 More about quantitative trait locus mapping with diallel designs. Genetical research 75: 243-7.

Sen, S., J. M. Satagopan, K. W. Broman, and G. A. Churchill, 2007 R/qtlDesign: inbred line cross experimental design. Mammalian genome 18: 87-93.

Sen, S., J. M. Satagopan, and G. A. Churchill, 2005 Quantitative trait locus study design from an information perspective. Genetics 170: 447-64.

Shorter, J. R., P. L. Maurizio, T. A. Bell, G. D. Shaw, D. R. Miller, et al., 2018 A diallel of the mouse collaborative cross founders reveals strong strain-specific maternal effects on litter size .

Simon, M. M., S. Greenaway, J. K. White, H. Fuchs, V. GailusDurner, et al., 2013 A comparative phenotypic and genomic analysis of C57BL/6J and C57BL/6N mouse strains. Genome biology 14: R82.

Srivastava, A., A. P. Morgan, M. L. Najarian, V. K. Sarsani, J. S. Sigmon, et al., 2017 Genomes of the mouse collaborative cross. Genetics 206: 537-556.

Tsaih, S.-W., L. Lu, D. C. Airey, R. W. Williams, and G. A. Churchill, 2005 Quantitative trait mapping in a diallel cross of recombinant inbred lines. Mammalian Genome 16: 344-355.

Turner, S. D., P. L. Maurizio, W. Valdar, B. S. Yandell, and P. W. Simon, 2018 Dissecting the Genetic Architecture of Shoot Growth in Carrot ( Daucus carota L.) Using a Diallel Mating Design. G3 (Bethesda, Md.) 8: 411-426.

Verhoeven, K. J. F., J.-L. Jannink, and L. M. McIntyre, 2006 Using mating designs to uncover QTL and the genetic architecture of complex traits. Heredity 96: 139-49.

Williams, R. W. and E. G. Williams, 2017 Resources for systems genetics. In Systems Genetics: Methods and Protocols, edited by K. Schughart and R. W. Williams, pp. 3-29, Springer New York, New York, NY.
Wood, A. R., T. Esko, J. Yang, S. Vedantam, T. H. Pers, et al., 2014 Defining the role of common variation in the genomic and biological architecture of adult human height. Nature genetics 46: 1173-86.

Yamamoto, A. H., 1994 Diallel analysis of temperature preference in Drosophila immigrans. Idengaku zasshi 69: 77-86.

Zhu, J. and B. S. Weir, 1996 Mixed model approaches for diallel analysis based on a bio-model. Genetical research 68: 233-40.

Zou, F., W. Sun, J. J. Crowley, V. Zhabotynsky, P. F. Sullivan, et al., 2014 A Novel Statistical Approach for Jointly Analyzing RNA-Seq Data from F1 Reciprocal Crosses and Inbred Lines. Genetics 197: 389-99.

\section{Appendix A: Prior specification}

Following the lead of (Lenarcic et al. 2012), conjugate priors were used for the parameters in the BayesDiallel model. For example, the strain-level additive effects are distributed following $a \sim$ $\mathrm{N}\left(0, \tau_{a}^{2}\right)$. For fixed effect terms, such as $\beta_{\text {inbred }}, \tau^{2}$ is set to $10^{3}$. For the variance parameters, consider $\sigma^{2}$ which is distributed following $\sigma^{2} \sim \operatorname{IG}(v / 2, \psi / 2)$. For the hyper parameters $v$ and $\psi 0.02$ and 2 were used, respectively. These represent diffuse priors, with the intention of allowing the information in the data to inform the estimates. The hyper parameter values can be adjusted within DIDACT R package. 